CANCER

\section{Antitumour metabolism}

Changing the way that tumours metabolize sugar can stall their growth.

When cells convert glucose into energy and molecular building blocks, the last step in the metabolic pathway is catalysed by an enzyme called pyruvate kinase. Dividing cells, such as cancer cells, contain a form of this enzyme called PKM2. Matthew Vander Heiden at the Massachusetts Institute of Technology in Cambridge and his colleagues found that small-molecule activators of PKM2 impaired tumour formation in mice bearing human cancer cells. Tumours developed later and remained smaller in treated mice than in untreated controls.

The activators bind to PKM2 at a previously unknown site. The authors suggest that, in so doing, the activators alter tumour metabolism in such a way as to deplete growth-promoting compounds produced earlier in the pathway.

Nature Chem. Biol. http://dx.doi. org/10.1038/nchembio. 1060 (2012)

NANOTECHNOLOGY

\section{Strong and stable nanocrystals}

A theoretical method could aid in the design of nanocrystalline metal alloys that retain their unusual properties, such as remarkable strength, at high temperatures.

Nanostructured metals, which are made up of individual metal grains less than 100 nanometres in size, are often unstable at high temperatures owing to instabilities arising from

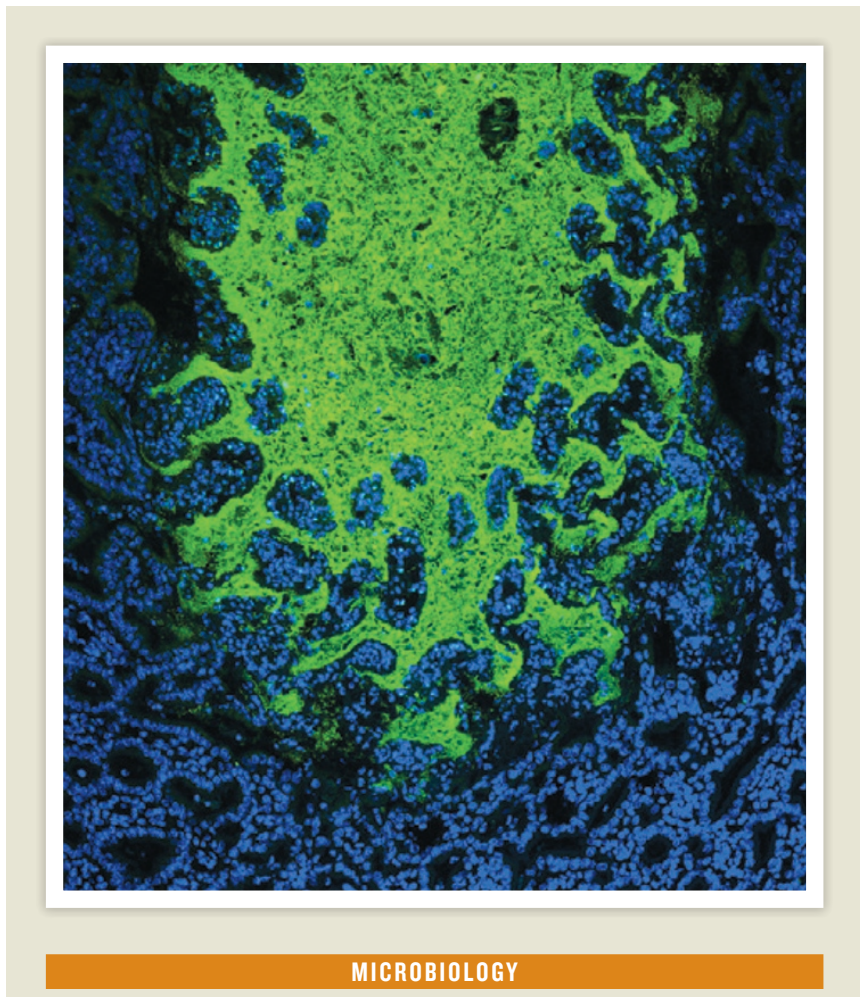

\section{Infection breaks truce}

Gastrointestinal infections can cause the immune system to attack some of the billions of microbes that are normally tolerated in the mammalian gut.

Yasmine Belkaid at the National Institute of Allergy and Infectious Diseases in Bethesda, Maryland, and her team demonstrated this immune response when they infected mice with the parasite Toxoplasma gondii. The infection caused the bacteria normally resident in the gut to breach the gut wall (pictured), activating the animals' immune systems. This triggered immune cells called $\mathrm{T}$ cells to target the gut bacteria. The $\mathrm{T}$ cells then developed into long-lasting memory $\mathrm{T}$ cells - providing an immunological record of the response.

As a result, a bout of food poisoning could have enduring consequences for the immune system, and could even contribute to the development of inflammatory bowel disease, the researchers suggest.

Science http://dx.doi.org/10.1126/science.1220961 (2012) the boundaries between grains. Christopher Schuh and his colleagues at the Massachusetts Institute of Technology in Cambridge developed a theoretical framework for predicting the energy associated with the grain boundaries. The researchers designed and produced an alloy of titanium and tungsten that turned out to be more stable at high temperatures than pure nanocrystalline tungsten, and that could be processed in bulk.

Science 337, 951-954 (2012)

\section{NEURODEVELOPMENT}

\section{Low-flow blood- vessel pruning}

The zebrafish brain's complex network of blood vessels develops not only through vessel growth, but also as a result of pruning, which is driven by changes in blood flow.

Jiu-lin Du at the Chinese Academy of Sciences in Shanghai and his team followed the development of transgenic zebrafish larvae in which cells of the blood vessels, blood and brain carry fluorescent tags. Over the course of six days after fertilization, the researchers imaged the creatures' developing midbrain. They found that, as the network of blood vessels expands, pruning occurs - mainly at loop-shaped segments when cells lining the vessels migrate towards adjacent unpruned segments, resulting in decreased vessel-network complexity. Pruned segments have a lower and more variable blood flow than unpruned ones, and experimental blockade of blood flow triggers the pruning process.

PLoS Biol. 10, e1001374 (2012)

$$
\text { EPIGENETICS }
$$

\section{Clues from baby blood spots}

Blood from the pricked heels of newborn babies can provide insight into the epigenome chemical changes to DNA that influence gene expression before diseases set in.

Most babies born in developed countries have drops of their blood blotted onto a slip of paper called a Guthrie card (pictured), which is used to screen for certain diseases. Vardhman Rakyan and David Leslie at 


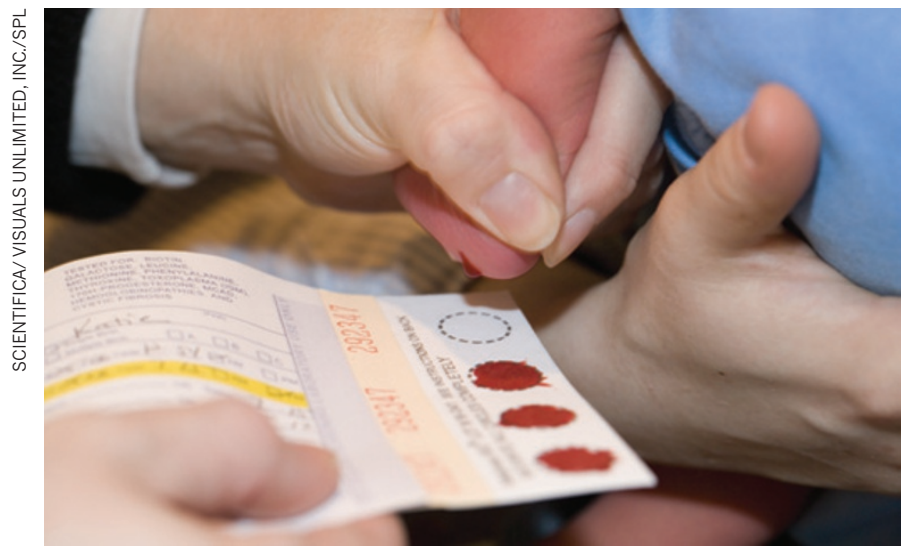

the Queen Mary University of London and their colleagues analysed DNA from the Guthrie cards of three healthy children born in 2000, looking for differences in patterns of DNA methylation, an epigenetic change that involves the addition of methyl groups to DNA. The researchers found about a dozen differences that were still present in samples taken three years later and did not seem to be linked to underlying genetic variation. This suggests that the changes might be the result of differences in conditions encountered in the womb.

Guthrie cards could help researchers to distinguish between epigenetic changes that are driving a disease and those that result from it. Genome Res. http://dx.doi.org/ 10.1101/gr.134304.111 (2012) For a longer story on this research, see http://go.nature.com/fgmz6w

\section{MEDICAL DEVICES}

\section{Smart way to seal cuts}

A 'smart' suture that doubles as a heater and a thermometer could promote wound healing.

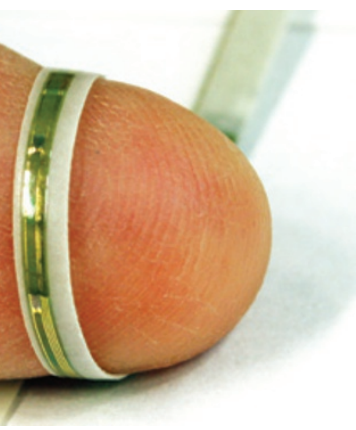

John Rogers of the University of Illinois at Urbana-Champaign and his colleagues have developed a prototype silicon-based suture (pictured) that is long, thin and flexible enough to be sewn into a wound. The suture contains circuit elements that can both heat tissue and sense its temperature. This enables the device to keep the wound at an ideal temperature for healing and to detect temperature increases associated with infection.

The researchers hope to develop sutures with other functions, such as programmable drug release. Small http://dx.doi.org/10.1002/ smll.201200933 (2012)

\section{PALAEONTOLOGY}

\section{Jagged jaws identify mollusc}

An analysis of the mouthparts of 505-million-year-old fossils suggests that the animals could be among the world's earliest molluscs.

Martin Smith of the

University of Toronto, Canada, analysed more than 300 fossils belonging to two early invertebrate groups, Odontogriphus and Wiwaxia, that have been variously classified as molluscs, annelids or a common ancestor. The fossils are from British Columbia's Burgess Shale, which provides a record of an explosion in new body forms that occurred around the Cambrian era (542 million to 488.3 million years ago).

The mouthparts of many of

COMMUNITY CHOICE

The most viewed papers in science

\section{Equations in papers $=$ fewer citations}

\section{HIGHLY READ \\ on www.pnas.org in July}

Biologists have a reputation for avoiding mathematics, and a study of citation rates shows that this is not misplaced.

Tim Fawcett and Andrew Higginson at the University of Bristol, UK, examined whether the inclusion of equations affected the citation rates of papers published in 1998 in three leading ecology and evolution journals. The authors found that papers received $28 \%$ fewer citations overall for each additional equation per page of the main text; for citations in experimental papers, this rose to $35 \%$.

The duo recommends that researchers use equations sparingly in their main article text to ensure that their ideas reach a wide audience.

Proc. Natl Acad. Sci. USA 109, 11735-11739 (2012)

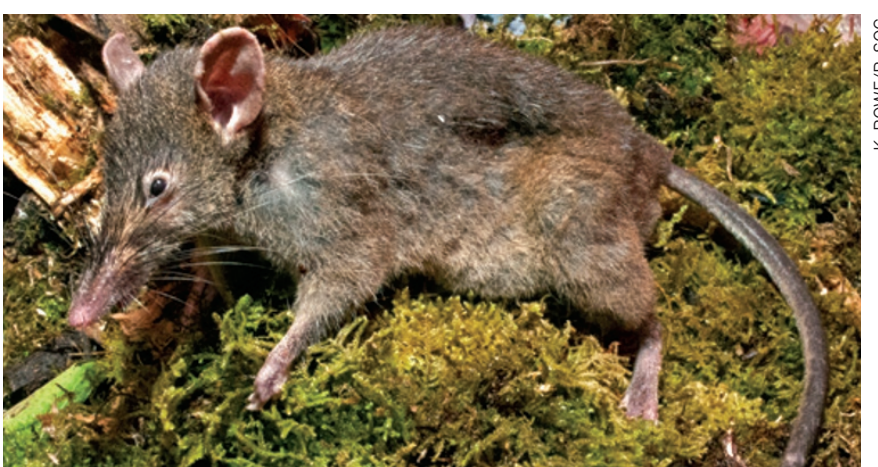

the fossils - rows of jagged teeth that rest on a grooved tongue - resemble those of modern-day molluscs. The teeth might have worked like a file, allowing the animals to grind soft tissues, the author suggests.

Proc. R. Soc. B http://dx.doi. org/10.1098/rspb.2012.1577 (2012)

\section{Rodent that cannot gnaw}

A shrew-rat that lacks molars and has oddly shaped incisors is an evolutionary anomaly among more than 2,200 known species of rodent.

Rats, mice and their kin owe their success in part to their ability to gnaw, which enables them to access foods that other mammals cannot.
Jacob Esselstyn of McMaster University in Hamilton, Ontario, and his colleagues report that the shrew-rat Paucidentomys vermidax (pictured) has a long, pointed face, incisors that differ in shape from those of other rodents, and no molars. These features could be adaptations that help the animal to find its favoured food - softbodied earthworms that do not need chewing. The loss of a previously successful evolutionary modification has allowed $P$. vermidax to take advantage of the available food in its forest home.

Biol. Lett. http://dx.doi. org/10.1098/rsbl.2012.0574 (2012)

\section{$\rightarrow$ NATURE.COM}

For the latest research published by Naturevisit:

www.nature.com/latestresearch 